\title{
Advances in Defocused-Beam Analysis and Compositional Mapping with the Electron-Probe Microanalyzer
}

\author{
P. K. Carpenter,* R. A. Zeigler,* and B. L. Jolliff* \\ *Department of Earth and Planetary Sciences and the McDonnell Center for the Space Sciences, Wash- \\ ington University in St. Louis, Campus Box 1169, Saint Louis, MO, 63130
}

Electron-probe microanalysis (EPMA) is fundamental to the study of lunar and meteoritic samples. Point analysis provides fine spatial chemical information, and large-scale mapping enables macroscale analysis of samples. Samples may contain submicron multiphase assemblages not resolved by the electron scattering volume of EPMA, and large area X-ray maps sample a significant number of phase boundaries and composite phases. Thus, both spot and pixel measurements are made on multiphase materials.

Improvements in $\Phi(\rho z)$ algorithms include more accurate analytical formulations of the $\Phi(\rho z)$ curve and more accurate treatment of the surface X-ray generation and emission quantity $\Phi_{0}$. The measured X-ray intensity in the sample relative to the standard is the k-ratio, and the calculated concentration $\mathrm{C}$ is determined by iterative calculation from $\mathrm{C}=\mathrm{k} * \mathrm{ZAF}$. While current microanalysis software uses a full $\Phi(\rho \mathrm{z})$ correction, the Bence-Albee (BA) $\alpha$-factor algorithm was originally used and calculated composition $\mathrm{C}$ as $\mathrm{C} / \mathrm{k}=\alpha+\mathrm{C}(1-\alpha)$, and applied to multielement systems via $\mathrm{C}=\mathrm{k} \beta$ where $\beta=\Sigma \mathrm{C} \alpha / \Sigma \mathrm{C}$ and was referenced relative to oxide standards [1]. Accuracy issues identified from use of constant term $\alpha$-factors have been eliminated by use of compositionally-dependent polynomial $\alpha$-factors (i.e., $\alpha_{1}+\alpha_{2} C+\alpha_{3} C^{2}$ ) that duplicate $\Phi(\rho z)$ algorithms for typical compositional systems [2]. The $\beta$ factor of this polynomial BA formulation is equivalent to the ZAF factor from a $\Phi(\rho z)$ algorithm and is referenced relative to elemental standards. This permits rapid and compact correction of data for metals and silicates.

EPMA provides a spatial resolution of $\sim 1 \mu \mathrm{m}$ in spot mode, but in defocused beam analysis (DBA) a spot size of 50-100 $\mu \mathrm{m}$ is used and the emission volume is a disk of diameter equal to the beam size and thickness equal to the X-ray production depth which is typically 1-3 $\mu \mathrm{m}$. Significant errors result if measurements of multiphase materials are processed using conventional correction algorithms that assume homogeneity in the scattering volume. The minerals in the analyzed volume have different densities and weight percent contribution to the bulk, but are sampled in terms of area or volume fraction by the electron beam. The chemical contrast between phases is important via the ZAF factors so the appropriate correction factors must be used for the phases in the analyzed volume. This requires knowledge of the phases in the volume and indeed image processing coupled with phase chemistry can provide the equivalent of DBA; however, all digital images sample phase boundaries and deconvolution of the signal is still necessary. With reference to analysis of homogeneous materials, it is necessary to know the phase composition for iterative correction, and the phase density for conversion from sampled area fraction to weight fraction of the phase. In DBA the contribution from each discrete phase is included as a weight fraction term in the iteration loop, and requires either explicit knowledge of the mineral chemistry or an approximation using a CIPW normative mineral calculation. The DBA technique and errors inherent in the calculation have been discussed previously [3-8]. DBA is ideal for a grain size larger than the primary electron scattering volume, and the primary emitted intensity is still obtained from discrete mineral phases. For grain size that is similar to the electron scattering volume (i.e., $\sim 1 \mu \mathrm{m}$ ), the roles of differential backscattering, $\mathrm{X}$ ray absorption and fluorescence are important.

An Excel spreadsheet has been constructed that uses Visual Basic for Applications macro code to perform the DBA correction. The procedure used for DBA correction follows the functional algorithm outlined by Albee et. al. $[4,5]$. In the algorithm, 1) the measured k-ratios from a DBA area are first corrected assum- 
ing a homogeneous material; 2) the concentrations obtained are next processed using a catanorm routine in order to obtain the normative phases that would be expected to form; 3 ) the weight percent normative minerals and their known $\beta$ factors are used to calculate a $\beta$ factor for the heterogeneous mixture of minerals which is weighted by the abundance of the normative mineral; 4) this heterogeneous $\beta$ factor is then used to correct the original k-ratio data to obtain a new estimate of concentration; 5) this new concentration is used to calculate the catanorm again; and 6) the process is iterated to conclusion. Generally it is observed that the most significant step occurs from homogeneous to initial normative mineralogy with relatively insignificant changes following that step. The advances in our DBA algorithm include the incorporation of the more accurate quantitative analysis procedures outlined above, coupled with the ability to modify the VBA code as needed to improve the normative algorithm. The normative phases are currently treated as fixed in composition but will be modified to address lunar mineralogy and also the ability to use actual mineral compositions for processing.

Two examples of DBA are shown in Table 1 and Fig 1. The first example is a hypothetical mixture comprised of equal area fractions of anorthite and an $\mathrm{En}_{80} \mathrm{Fs}_{20}$ pyroxene. The ideal weight percent analysis was calculated from the known area fraction and density values for the two phases. The k-ratios for anorthite and the pyroxene were assumed to vary linearly with area and were input in the DBA correction using the ZAF factors for anorthite and pyroxene. The k-ratio data was also used to calculate an analysis for an assumed homogeneous sample. DBA of Meteorite Hills (MET) 01210 is illustrated in Fig. 1 which shows a symplectite intergrowth of fayalite (bright), hedenbergite (intermediate), and silica (dark) [9]. Using the conventional correction on multiphase materials typically results in overcorrection that is applied to an assumed elemental mixture that is in reality segregated into discrete phases. Note the data for $\mathrm{Mg}$ and $\mathrm{Fe}$ in the two examples are corrected in opposite directions due to the composition of the phases considered.

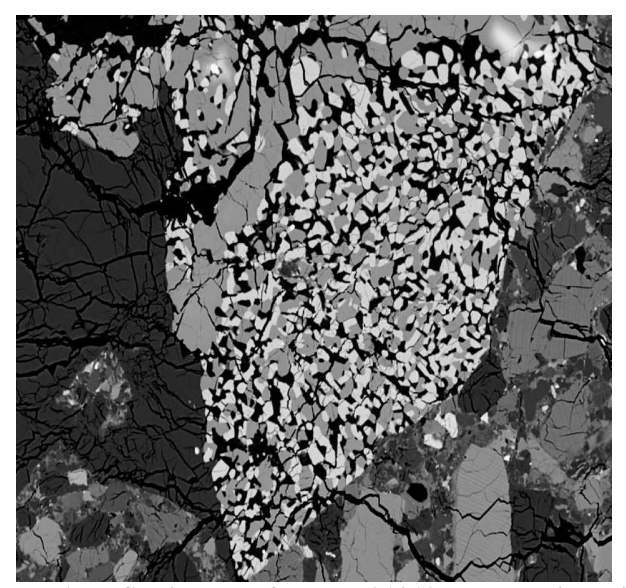

Table 1.

\begin{tabular}{|c|c|c|c|c|c|c|}
\hline & $\begin{array}{c}\text { AE } \\
\text { Ho }\end{array}$ & $\begin{array}{c}\text { AE } \\
\text { DBA }\end{array}$ & $\begin{array}{c}\text { Beta } \\
\text { Ho/Ht }\end{array}$ & $\begin{array}{c}\text { Symp. } \\
\text { Ho }\end{array}$ & $\begin{array}{c}\text { Symp. } \\
\text { DBA }\end{array}$ & $\begin{array}{c}\text { Beta } \\
\text { Ho/Ht }\end{array}$ \\
\hline $\mathrm{Mg}$ & 8.60 & 9.09 & 0.95 & 1.17 & 0.95 & 1.18 \\
\hline $\mathrm{Al}$ & 10.45 & 9.45 & 1.11 & 0.27 & 0.22 & 1.17 \\
\hline $\mathrm{Si}$ & 22.63 & 22.66 & 1.01 & 22.87 & 22.18 & 0.99 \\
\hline $\mathrm{Ca}$ & 6.98 & 7.02 & 1.00 & 3.73 & 3.74 & 0.96 \\
\hline $\mathrm{Ti}$ & & & & 0.22 & 0.20 & 1.07 \\
\hline $\mathrm{Fe}$ & 5.09 & 5.07 & 1.01 & 31.46 & 31.17 & 0.97 \\
\hline $\mathrm{O}$ & 46.24 & 46.71 & 0.99 & 39.80 & 41.52 & 0.92 \\
\hline $\mathrm{Key}$ 1 1 and 2. An-En & \\
processed assuming single homogeneous phase (1) vs. DBA (2) \\
3. Ratio of $\beta$ factor for homogeneous vs. DBA for An-En ${ }_{80} \mathrm{Fs}_{20}$. \\
4 and 5. MET 01210 symplectite analysis processed assuming \\
homogenous phase (4) vs DBA (5). \\
6. Ratio of $\beta$ factor for homogeneous vs. DBA for MET 01210. \\
\hline
\end{tabular}

Figure 1 BSE image of MET 01210 symplectite with fayalite (bright), hedenbergite (medium), and silica (dark). 30um spot used for DBA. Width of field $300 \mathrm{um}$.

\section{References}

[1] Bence, A.E., and Albee, A. L. (1968) J. Geology, Vol. 76, p. 382-403.

[2] Armstrong, J. T. (1988) Microbeam Analysis, 469-476.

[3] Warren, P. (1997) LPS XXVIII, 1497-1498.

[4] Albee, A. L. et al. (1977) $8^{\text {th }}$ Int. Congr. Xray Opt. 526-537

[5] Albee, A. L. et al. (1977) LPS VIII, 7-9.

[6] Nazarov, M. A. et al. (1982) LPS XIII, 582-583.

[7] Lindstrom, D. J. (1999) LPS XXX, No. 1917

[8] Berlin, J. (2008) Microsc. Microanal. 14 (Suppl 2) 110-111.

[9] Zeigler et. al. (2005) LPS XXXVI (2005), \#2385.

[10] Jolliff, B. L. (2008) LPS XXXIX, \#2519. 